\title{
Towards Large-Scale Steady-State Enhanced Nuclear Magnetization with In Situ Detection
}

\author{
John W. Blanchard, ${ }^{*},+\ddagger$ Barbara Ripka, "Benjamin A. Suslick, ${ }^{\S} \|$ Dario Gelevski, ${ }^{\S}$ \\ Teng Wu, ${ }^{\dagger, \perp}$ Kerstin Münnemann, $\#, \Phi$ Danila Barskiy, ${ }^{*, \dagger, \perp}$ and Dmitry \\ Budker ${ }^{\perp, \dagger, @ ~}$ \\ $\dagger$ †elmholtz-Institut Mainz, GSI Helmholtzzentrum für Schwerionenforschung, 55128 \\ Mainz, Germany \\ $\ddagger$ NVision Imaging Technologies GmbH, 89081 Ulm, Germany \\ \Max Planck Institute for Polymer Research, 55128 Mainz, Germany \\ $\S$ Department of Chemistry, University of California, Berkeley, CA 94720-3220, USA \\ \|Department of Chemistry and Beckman Institute for Advanced Science and Technology, \\ University of Illinois at Urbana-Champaign, Urbana, IL 61801 USA \\ $\perp$ Johannes Gutenberg-Universität Mainz, 55128 Mainz, Germany \\ \# Technische Universität Kaiserslautern, 67663 Kaiserslautern, Germany \\ @ Department of Physics, University of California, Berkeley, California 94720, USA
}

E-mail: blanchard@uni-mainz.de; dbarskiy@uni-mainz.de

\section{Abstract}

Signal Amplification By Reversible Exchange (SABRE) boosts NMR signals of various nuclei enabling new applications spanning from magnetic resonance imaging to analytical chemistry and fundamental physics. SABRE is especially well positioned for continuous generation of enhanced magnetization on a large scale, however, several challenges need to be addressed for accomplishing this goal. Specifically, SABRE requires (i) a specialized catalyst capable of reversible $\mathrm{H}_{2}$ activation and (ii) physical transfer of the sample from the point of magnetization generation to the point of detection (e.g., a high-field or a benchtop NMR spectrometer). Moreover, (iii) continuous parahydrogen bubbling accelerates solvent (e.g., methanol) evaporation, thereby limiting the experimental window to tens of minutes per sample.

In this work, we demonstrate a strategy to rapidly generate the best-to-date precatalyst (a compound that is chemically modified in the course of the reaction to yield the catalyst) for SABRE, [Ir(IMes)(COD)Cl] (IMes $=1,3$ bis-(2,4,6-trimethylphenyl)-imidazol-2-ylidene, $\mathrm{COD}=$ cyclooctadiene) via a highly accessible synthesis. Second, we measure hyperpolarized samples using a home-built zero-field NMR spectrometer and study the field dependence of hyperpolarization directly in the detection apparatus, eliminating the need to physically move the sample during the experiment. Finally, we prolong the measurement time and reduce evaporation by presaturating parahydrogen with the solvent vapor before bubbling into the sample. These advancements extend opportunities for exploring SABRE hyperpolarization by researchers from various fields and pave the way to producing large quantities of hyperpolarized material for long-lasting detection of SABRE-derived nuclear magnetization.

\section{Introduction}

Hyperpolarization techniques increase nuclear magnetic resonance (NMR) signals by 
orders of magnitude. This tremendous signal enhancement enables various applications in biomedicine, ${ }^{1-5}$ analytical chemistry, ${ }^{6-8}$ catalysis, ${ }^{9,10}$ and fundamental physics. ${ }^{11-13}$

One of the newest hyperpolarization techniques is signal amplification by reversible exchange (SABRE), ${ }^{14,15}$ a physicochemical approach based on the use of parahydrogen (para$\mathrm{H}_{2}$ ), a nuclear spin isomer of the hydrogen molecule. ${ }^{16}$ While para- $\mathrm{H}_{2}$ itself is NMR-silent (total nuclear spin $I=0$ ), its latent nuclear spin order can be converted into observable magnetization using organometallic magnetization-transfer catalysts. ${ }^{17}$ Such catalysts facilitate bilateral exchange of a substrate to be polarized with para- $\mathrm{H}_{2}$, thus enabling nuclear spin-order conversion from para- $\mathrm{H}_{2}$ to the substrate. $^{18}$

SABRE is fast, readily scalable, and does not require high magnetic fields for generating polarization, etc. - it is thus a promising approach to produce large quantities of hyperpolarized material. Large-scale hyperpolarization would be useful for imaging chemical reactors ${ }^{19}$ or for applications in fundamental physics. For example, CASPEr-gradient is a dark matter search in which a large volume of nuclear spin-polarized material is required to transduce dark matter fields into detectable magnetic signals. ${ }^{11}$

Despite being scalable in principle, SABRE has practical limitations that hinder achieving and detecting long-lasting hyperpolarization on a large scale. First, SABRE requires a catalyst produced by activating a precatalyst compound which is not readily available commercially. One of the most often used precatalysts for SABRE is [Ir(IMes)(COD)Cl] (1), where IMes is 1,3-bis-(2,4,6-trimethylphenyl)imidazol-2-ylidene, and COD is cyclooctadiene. ${ }^{20}$ This organometallic complex is synethesied under inert atmosphere (either in a glovebox or using Schlenk lines), and therefore it is not universally accessible for researchers outside of a specialized chemical laboratory. Second, since the build-up of enhanced magnetization in SABRE is achieved at low fields (either at mT-range for polarizing ${ }^{1} \mathrm{H}$ spins ${ }^{18,21}$ or at sub- $\mu \mathrm{T}$-range for polarizing heteronuclei ${ }^{22,23}$ ), samples need to be transferred to much larger fields (several tesla) of NMR spectrometers for signal detection. This is typically performed by physically moving the sample (either by hand, or using shuttling setups ${ }^{24}$ ) from the point of para $-\mathrm{H}_{2}$ bubbling to the spectrometer. The requirement of moving the sample from the point of magnetization generation to the point of the detection is a clear disadvantage for detecting large-scale SABRE-derived magnetization, because it imposes engineering limitations on the experimental setup and introduces sources of signal attenuation. Finally, continuous and often intense para- $\mathrm{H}_{2}$ bubbling through the sample rapidly evaporates the solvent (typically, methanol) limiting the experimental window to several tens of minutes ${ }^{25}$ per sample before changes in the sample composition begin to affect signal enhancement.

Herein, we demonstrate approaches to resolve all the above-mentioned problems bringing one step closer the detection of large-scale boluses of steady-state SABRE-derived hyperpolarization.

First, we demonstrate a strategy of rapid (under five minutes) and accessible (under ambient atmosphere) synthesis of $\mathbf{1}$, to date, the most popular SABRE precatalyst. The use of an inert atmosphere is not necessary because the reaction between readily available precursors, $[\operatorname{Ir}(\mathrm{COD}) \mathrm{Cl}]_{2}$ and IMes, happens within one to three minutes after mixing with the reaction yield of $82 \%$. In addition, we employ a strategy of generating reactive carbene IMes in situ by reacting IMes. $\mathrm{HCl}$ with $\mathrm{K}_{2} \mathrm{CO}_{3}$ in acetone with $85 \%$ yield of the desired product. This strategy uses stable compounds significantly simplifying handling the precursors and eliminating the need for sophisticated chemical equipment.

Second, we detect SABRE-hyperpolarized molecules using zero- to ultralow-field (ZULF) NMR spectrometer based on a home-built optically pumped magnetometer (OPM). Detection of NMR signals using commercially-available OPMs has also been demonstrated. ${ }^{26,27}$ Detecting magnetization continuously at the point of its production obviates the need of physically moving the sample, thus, providing opportunities for straightforward scaling as well as in situ analysis of magnetic field dependence of hyper- 
polarized chemical signals. We carry out such analysis for ${ }^{15} \mathrm{~N}$-pyridine at near-zero magnetic field and reproduce the expected magnetization sign change. ${ }^{28}$

Third, we present a simple approach that significantly reduces solvent evaporation. This approach is based on presaturating the para- $\mathrm{H}_{2}$ gas with the solvent (methanol) vapor prior to bubbling through the SABRE solution. This allowed us to detect SABRE magnetization continuously on the same sample by ZULF NMR for a period of five hours.

Taken together, these advancements significantly extend capabilities of SABRE and pave the way to long-lasting steady-state hyperpolarization on the large scale for applications in various scientific disciplines.

\section{Results and discussion}

\section{Facile Catalyst Synthesis}

Cowley et al. showed that $[\operatorname{Ir}(\mathrm{COD})(\mathrm{IMes}) \mathrm{Cl}]$ (1) is the precursor of the best-to-date SABRE magnetization transfer catalyst. ${ }^{20}$ Indeed, upon activation under an $\mathrm{H}_{2}$ atmosphere and with excess substrate, $\mathbf{1}$ transforms into the hexacoordinated hydride complex $\left[\mathrm{Ir}(\mathrm{IMes}) \mathrm{S}_{3} \mathrm{H}_{2}\right] \mathrm{Cl}$ (2), where $\mathrm{S}$ is a substrate (e.g., pyridine). Complex 2 exhibits optimal exchange parameters for SABRE (Scheme 1A). ${ }^{29}$ Typically, $\mathrm{H}_{2}$ activation occurs in an NMR tube followed by SABRE experiments on the substrate. To date, $\mathbf{2}$ is the best-known SABRE catalyst, mainly due to the optimal exchange parameters and relatively large separation of hydride protons from other magnetic nuclei in the complex which extends their relaxation times. ${ }^{15}$

Compound 1 belongs to a family of organometallic complexes with $N$-heterocyclic carbene (NHC) ligands. These compounds possess unique catalytic and biological activity due to the electronic and steric properties of NHC ligands. ${ }^{30}$

First reports of the synthesis of the compounds similar to 1 date back to early 2000-s. Nolan et al. demonstrated that NHC SIMes reacts with $\left[\operatorname{Ir}(\mathrm{COD})(\mathrm{Py})_{2}\right] \mathrm{PF}_{6}$ to produce

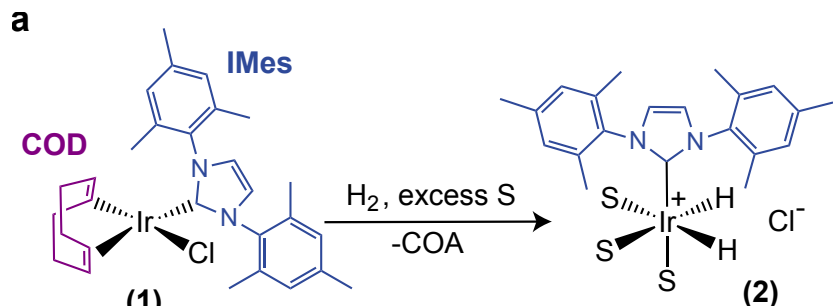

(1)

(2)

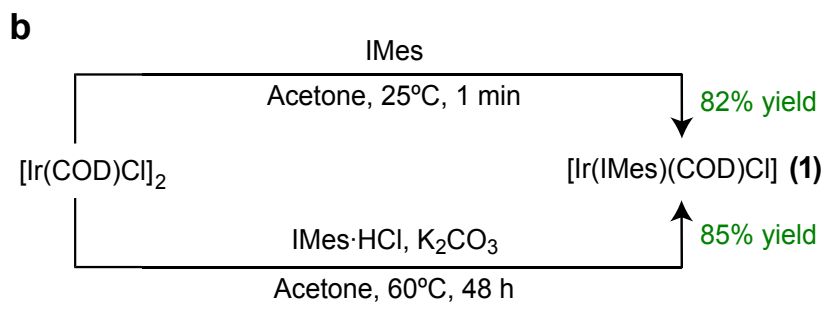

Scheme 1: A) Activation of the organometallic complex [Ir(IMes)(COD)Cl] (1) under an $\mathrm{H}_{2}$ atmosphere and with excess substrate $\mathrm{S}$ (e.g., pyridine) which produces magnetizationtransfer catalyst $\left[\mathrm{Ir}(\mathrm{IMes}) \mathrm{S}_{3} \mathrm{H}_{2}\right] \mathrm{Cl}(\mathbf{2})$. B) Synthesis of $\mathbf{1}$ under ambient atmosphere.

$[\operatorname{Ir}(\mathrm{COD})(\mathrm{SIMes})(\mathrm{Py})] \mathrm{PF}_{6} \cdot{ }^{31}$ Vazquez-Serrano et al. in 2006 synthsized $\mathbf{1}$ for the first time. ${ }^{32}$ In general, the synthetic procedure is straightforward and consists of one reaction from the available precursors, $[\operatorname{Ir}(\mathrm{COD}) \mathrm{Cl}]_{2}$ and IMes. However, all procedures reported to date require inert atmospheres realized either in a glove-box or in Schlenk lines taking into account reactive nature of NHCs.

A typical synthetic procedure consists of weighing the reagents under air, brief transportation into the flask, substituting the air with inert gas (argon or $\mathrm{N}_{2}$ ) and stirring the mixture for more than 20 hours. ${ }^{32}$ With an initial goal to monitor reaction kinetics under inert atmosphere, we took $0.1 \mathrm{~mL}$ aliquots of the reaction mixture containing $60 \mathrm{mg}$ of iridiumcompound dimer $[\operatorname{Ir}(\mathrm{COD}) \mathrm{Cl}]_{2}$ and $60 \mathrm{mg}$ of IMes in $3 \mathrm{~mL}$ of benzene every 20 minutes. These aliquots were used for ${ }^{1} \mathrm{H}$ NMR analysis. Remarkably, ${ }^{1} \mathrm{H}$ NMR signals of $\mathbf{1}$ were clearly visible even from the first aliquot and their amplitude was not significantly changing for the aliquotes taken at longer reaction times. This demonstrates that the synthesis of (1), in fact, happened in the first minute after mixing the reagents. Verifying the reaction 


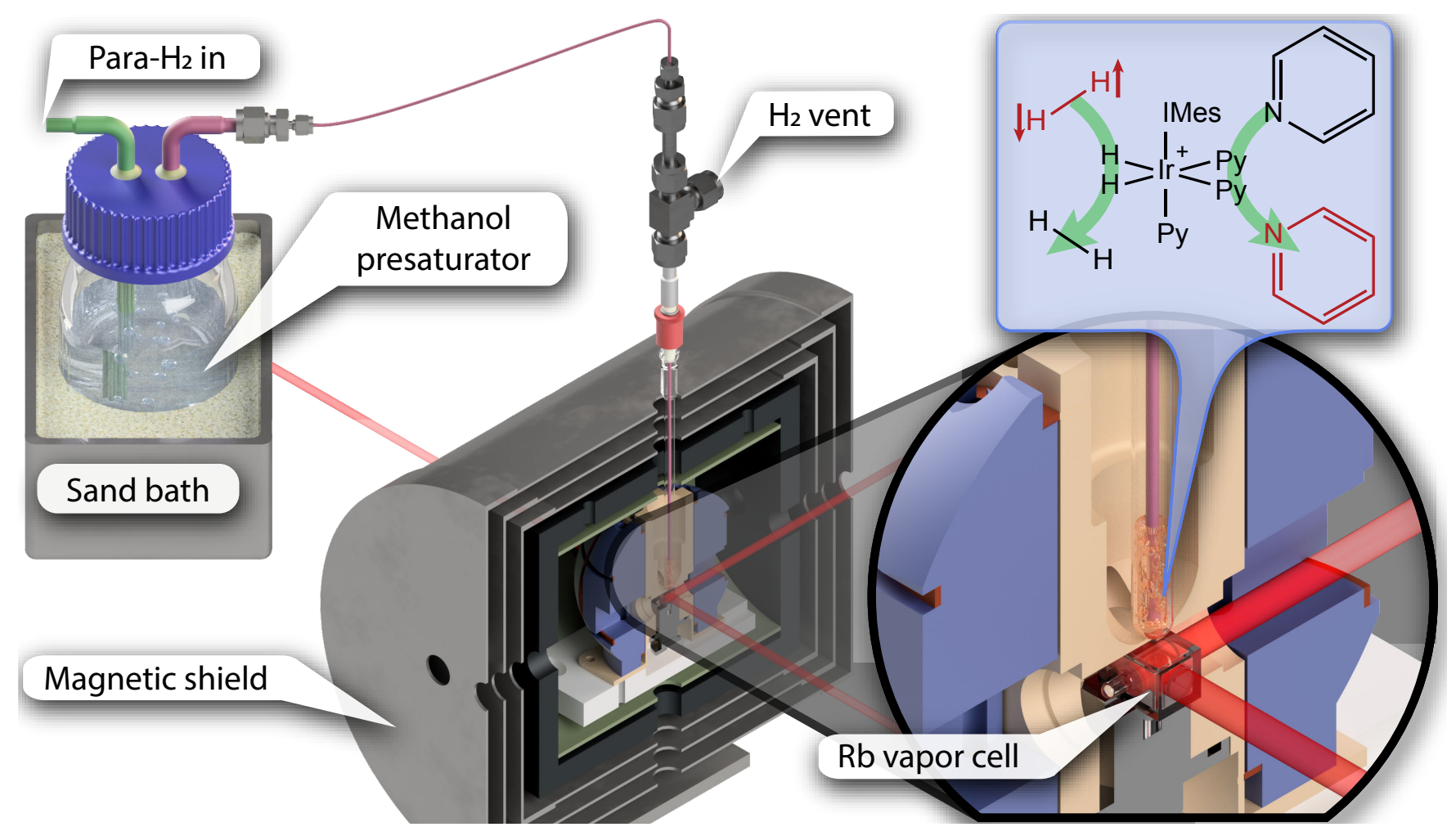

Figure 1: Experimental setup for SABRE-SHEATH for ZULF NMR. First, para- $\mathrm{H}_{2}$ is bubbled through methanol presaturator that prevents rapid evaporation of the SABRE sample located in the ZULF NMR spectrometer. The inset shows the SABRE reaction with pyridine as a substrate.

conditions in less toxic solvents demonstrated that acetone provides a similar reaction rate. Since the reaction is fast, keeping it for more than 20 hours in the reaction flask is not necessary. Indeed, synthesis carried out with a bigger batch of the reagents $(0.5 \mathrm{~g}$ of IMes and $0.5 \mathrm{~g}$ of $[\mathrm{IrCl}(\mathrm{COD})]_{2}$ in $10 \mathrm{~mL}$ of acetone) demonstrated excellent yield of $>80 \%$ while the total mixing time took less than five minutes (Scheme 1b).

Taking into account the fact that availability of the free carbene and its storage in the absence of inert atmosphere can be an issue, we adopted the procedure demonstrated by Savka et al. ${ }^{33}$ (Scheme 1b). In this approach, IMes is replaced by the corresponding salt IMes. $\mathrm{HCl}$ and the reaction is carried out at elevated temperature $\left(60{ }^{\circ} \mathrm{C}\right)$ in the presence of base $\mathrm{K}_{2} \mathrm{CO}_{3}$. This approach also showed excellent yield (85\%) but required longer stirring: high reaction yield was found after about 48 hours. Purification was achieved after passing the crude reaction mixture through a short pad of silica and removing residual solvent under re- duced pressure.

\section{In Situ Detection}

Zero- to ultralow-field (ZULF) NMR enables distinguishing chemicals in the liquid state based on their nuclear spin-spin couplings and topologies. ${ }^{34,35}$ Given the fact that achieving homogeneity is easier at zero field than at high field, ZULF NMR is a suitable technique for distinguishing molecules in liquid state and detecting and characterizing weak physical interactions. While ZULF NMR measurements can be performed on samples initially prepolarized by placing them in a permanent magnet before the measurement, ${ }^{36}$ the downside of this approach is that the sample has to be shuttled. This hinders measurements during and shortly after prepolarization and limits polarization values to only $\sim 10^{-6}$, insufficient for many problems at hand. Therefore, a combination of high and long-lasting nuclear spin polarization detected in situ by ZULF NMR is of great interest. 
a

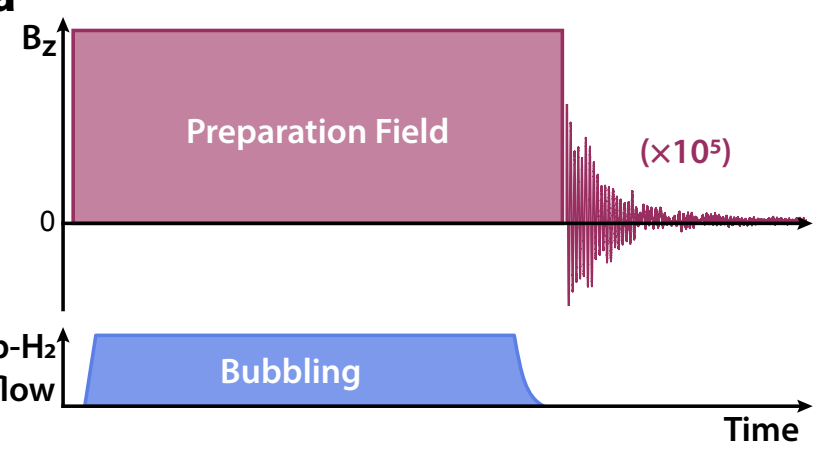

b

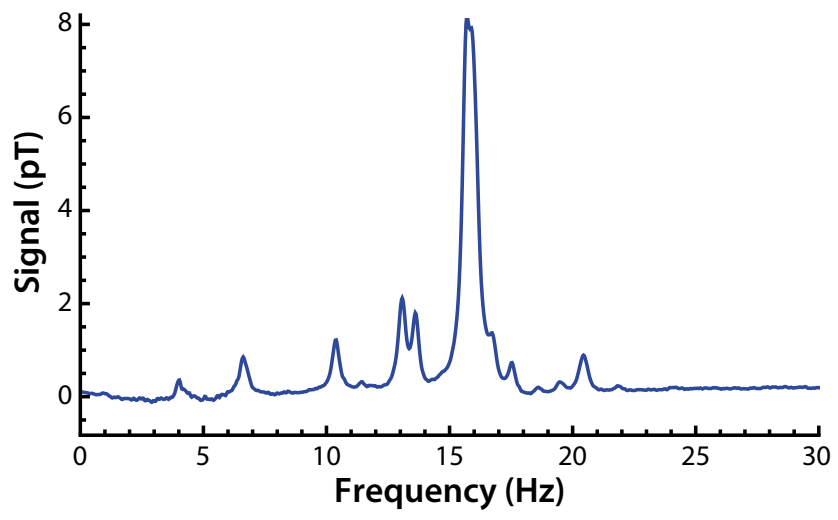

C

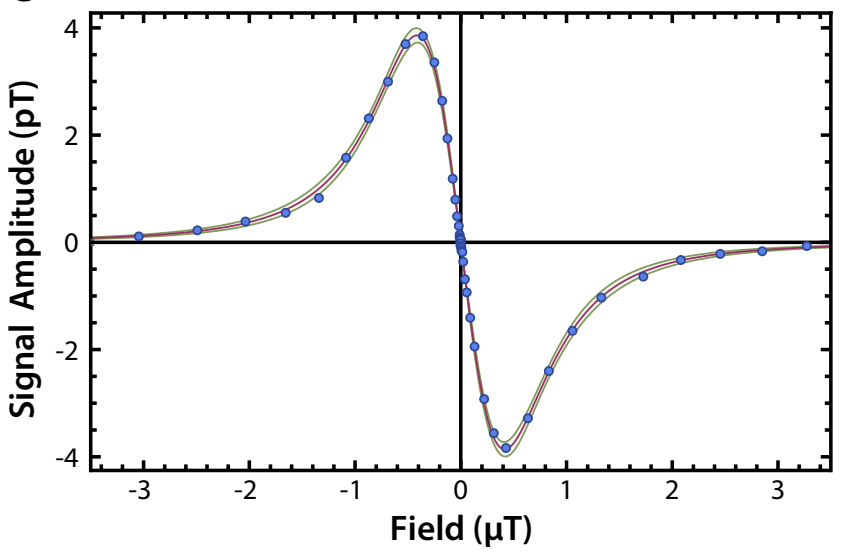

Figure 2: a) The ZULF NMR experimental sequence consisting of para- $\mathrm{H}_{2}$ bubbling with a magnetic field turned on, abrupt switching off of the field, and subsequent signal acquisition with the optically pumped magnetometer (OPM). b) ZULF NMR $J$-spectrum of SABRE-polarized $\left[{ }^{15} \mathrm{~N}\right]$-pyridine, acquired using the method described above. c) Dependence of the ZULF NMR signal amplitude on the magnetic field. Two absorptive Lorentzians with opposite signs were fitted to the results; $5 \sigma$ confidence bands are shown in yellow.
A low-field version of SABRE dubbed SABRE-SHEATH (SABRE in SHield Enables Alignment Transfer to Heteronuclei ${ }^{1}$ ) was recently developed. ${ }^{22,23}$ In SABRE-SHEATH, polarization transfer from para- $\mathrm{H}_{2}$-derived protons to ${ }^{15} \mathrm{~N}$ spins takes place at submicrotesla magnetic fields. While the detection of ZULF NMR signals originating from SABRE-polarized samples was demonstrated previously, ${ }^{37}$ it relied on the singlet-order transfer from para $-\mathrm{H}_{2}$ to $\left[{ }^{15} \mathrm{~N}\right]$-pyridine, thus, no magnetic field was applied during para $-\mathrm{H}_{2}$ bubbling through the sample. In order for the para- $\mathrm{H}_{2}$-derived singlet order to be converted to heteronuclear magnetization, a state-mixing magnetic field of $0.3-1.0 \mu \mathrm{T}$ needs to be applied during the para $-\mathrm{H}_{2}$ bubbling process. ${ }^{15,28}$ Here we study the magnetic field dependence of the hyperpolarized $\left[{ }^{15} \mathrm{~N}\right]$-pyridine signals in situ.

The mechanism of the SABRE reaction with pyridine substrate is depicted in Fig. 1. Experimental setup consists of a gas line with options to supply either para- $\mathrm{H}_{2}$ for the SABRE experiments or nitrogen for purging. First, para$\mathrm{H}_{2}$ gas is bubbled through methanol heated to $\sim 65^{\circ} \mathrm{C}$ in the presaturator bottle and then through the SABRE sample. Presaturation is described in more details in the next section. Signal acquisition is performed with a rubidium-vapor magnetometer placed inside the ZULF shield.

A measurement follows the scheme shown in Fig. 2a and is repeated for different values of the magnetic field applied in the direction collinear with the sensitive axis of the magnetometer. In the beginning, para $-\mathrm{H}_{2}$ is bubbled though the sample for $15 \mathrm{~s}$. The field and the bubbling are then turned off and the acquisition of the free decay (FD) signal is performed over $10 \mathrm{~s}$. Then the next measurement is recorded in which the value of the magnetic field is incremented; details of the experiments are given in the Supporting Information (SI). The total duration from the beginning of bubbling until the end of the measurement was $30 \mathrm{~s}$. The described procedure allows to detect SABRE-

\footnotetext{
${ }^{1}$ The name is a slight misnomer because it is nuclear orientation and not alignment that is transferred.
} 
enhanced signals of pyridine without the necessity of physically removing the sample from the site of polarization generation. Figure $2 \mathrm{~b}$ shows the ZULF NMR $J$-spectrum of $\left[{ }^{15} \mathrm{~N}\right]$-pyridine. ${ }^{37}$

Sample composition for SABRE experiments was optimized to achieve maximal signal-tonoise ratio (SNR), see SI. The optimal composition was $1.9 \mathrm{M}$ pyridine in methanol (15 vol\%) with $25 \mathrm{mM}$ catalyst. Since only ${ }^{15} \mathrm{~N}$-labeled molecules contribute to the signal, 0.04 M $\left[{ }^{15} \mathrm{~N}\right]$ pyridine was added to increase the SNR. The optimal pressure within the possibilities of the para $-\mathrm{H}_{2}$ generator was found to be at 6-7 bar with the maximum possible flow to keep this pressure stable. The best SNR was achieved at a sample temperature of $37^{\circ} \mathrm{C}$ and a bubbling duration of $15 \mathrm{~s}$ with a $1 \mathrm{~s}$ delay between the bubbling cessation and the start of the signal acquisition.

In order to study the magnetic-field dependence of the polarization transfer during in situ ZULF-SABRE experiments, a variable magnetic field in the range of $-3 \mu \mathrm{T}$ to $3 \mu \mathrm{T}$ was applied during the bubbling. A plot of the maximum signal amplitude over the applied magnetic field strength is shown in Fig. 2c. Two absorptive Lorentzians with opposite signs were fitted to the data points; $5 \sigma$ confidence bands are shown in yellow.

Since the abrupt switching off of the magnetic field does not convert the singlet spin order into observable magnetization, this protocol measures only the magnetization which was transferred to $\left[{ }^{15} \mathrm{~N}\right]$-pyridine at the applied field. The results are similar to those obtained by Colell et al. ${ }^{28}$ for SABRE-SHEATH of $\left[{ }^{15} \mathrm{~N}\right]$-acetonitrile carried out using sample transfer to high field for detection.

\section{Long-lasting Signal Acquisition}

Results of a long-lasting in situ SABRESHEATH experiment are shown in Fig. 3. For this experiment, no magnetic field was applied during para $-\mathrm{H}_{2}$ bubbling. Immediately after bubbling cessation, a pulse of amplitude $B$ and duration $t_{\mathrm{p}}$ such that $\left(\gamma_{\mathrm{H}}-\gamma_{\mathrm{N}}\right) B t_{\mathrm{p}}=\pi / 2$ was applied in the direction of the magnetometer sensitive axis to convert singlet order that was
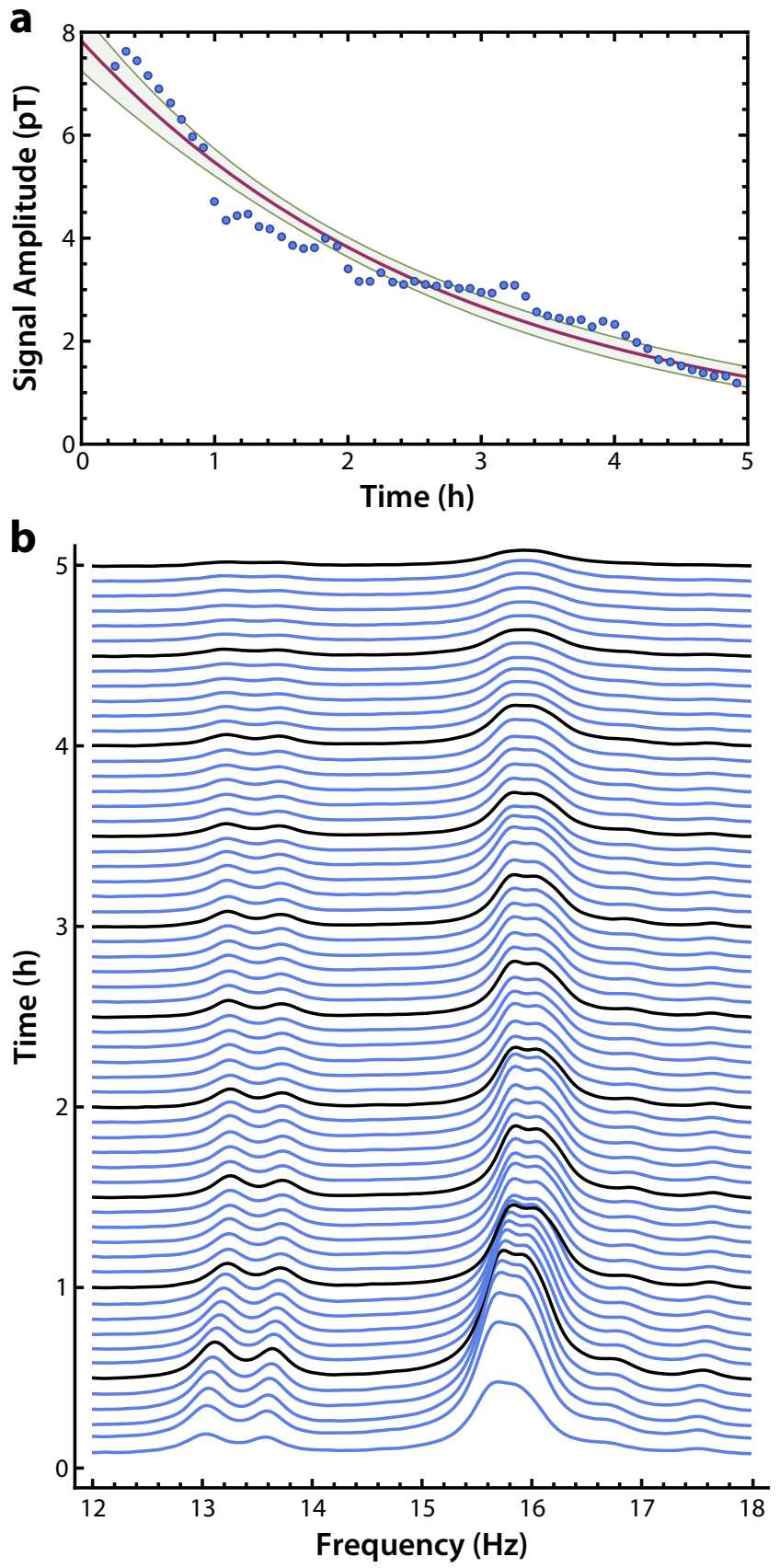

Figure 3: Results of a continuous long-time in situ SABRE-ZULF experiment. The signal was continuously detected for over five hours. a) The maximum signal amplitude is plotted over time. A decay occurs due to a changing substrate concentration since the methanol solvent gradually evaporates. An exponential fit gives a decay constant of $T_{\text {evap }}=(2.79 \pm 0.10)$ hours. 
transferred to $\left[{ }^{15} \mathrm{~N}\right]$-pyridine at zero field into evolving magnetization. Here, $\gamma_{\mathrm{H}}$ and $\gamma_{\mathrm{H}}$ are the gyromagnetic ratios of ${ }^{1} \mathrm{H}$ and ${ }^{15} \mathrm{~N}$, respectively. SABRE-enhanced ZULF NMR spectra of $\left[{ }^{15} \mathrm{~N}\right]$-pyridine were recorded every $30 \mathrm{~s}$ for more than $5 \mathrm{~h}$. Over time, the signal amplitude decreased with an exponential decay constant of $T_{\text {evap }}=(2.79 \pm 0.10) \mathrm{h}$ (Fig. 3a). This signal decrease could be explained by an increase of pyridine concentration in the sample solution while methanol evaporates which, in turn, diminishes the efficiency of polarization transfer. ${ }^{15}$ The gradually changing sample composition also influences $\left[{ }^{15} \mathrm{~N}\right]$-pyridine $J$-couplings, ${ }^{38,39}$ which is clearly visible as frequencies shift in the recorded ZULF NMR spectra (Fig. 3b).

In our setup, without a methanol presaturator the solvent evaporates fully in about 40 minuntes; therefore, the presaturator prolongs the measurement time by an order of magnitude. Sample evaporation may be further diminished or even avoided by cooling the upper part of the sample tube, letting methanol recondense inside the sample container. Alternatively, one could consider replacing methanol with a less-volatile solvent, such as water, after activating the catalyst.[refs] We note that the observed decay in signal intensity is not due to decreasing sample volume, since the total sample volume exceeded the magnetometer sensitive volume throughout the whole experiment, and is instead a result of the changes in sample composition (i.e., pyridine concentration).

\section{Conclusions}

In this work, we demonstrated strategies of overcoming the challenges facing SABRE as a tool for generating large-scale long-lasting in situ detected hyperpolarization. A facile synthesis of the most widely used SABRE precatalyst, $[\mathrm{Ir}(\mathrm{IMes})(\mathrm{COD}) \mathrm{Cl}]$ is reported under ambient atmosphere without using sophisticated chemical equipment. We performed measurements of ZULF NMR $J$-spectra of $\left[{ }^{15} \mathrm{~N}\right]$ pyridine and measured in situ the magnetic field profile of the polarization transfer. We ad- ditionally extended the capabilities of detecting the SABRE-SHEATH signal by an order of magnitude in time by presaturating para- $\mathrm{H}_{2}$ gas with solvent vapor before bubbling through the SABRE sample. This allowed us to measure SABRE signals continuously in situ via ZULF NMR for more than $5 \mathrm{~h}$.

Performing SABRE experiments with in situ ZULF NMR detection significantly increases experimental reproducibility by eliminating the requirement of physically removing the sample from the site of polarization generation. Moreover, continuous in situ experiments enable optimization of experimental parameters. The presented results provide a solution to challenging problems facing SABRE as a method for producing long-lasting steady-state nuclear spin magnetization on a large scale. We anticipate applications in biomedicine, analytical chemistry, and fundamental physics.

Acknowledgement This research was supported by the DFG Koselleck Program and the Heising-Simons and Simons Foundations, the European Research Council under the European Union's Horizon 2020 Research and Innovative Programme under Grant agreement No. 695405 (J.W.B., T.W. and D.B.), as well as by the Max Planck Graduate Center, Mainz. We thank Peter Blümler for discussing the idea of saturating parahydrogen gas with methanol vapor in order to reduce sample evaporation and Gary Centers for designing and maintaining the temperature-control system for the ZULF NMR spectrometer. D.A.B. acknowledges support from Alexander von Humboldt Foundation in the framework of the Sofja Kovalevskaja Award.

\section{Supporting Information Avail- able}

Detailed synthetic procedure, optimization of reaction parameters, acquisition sequence information for all presented experiments, details on experimental methods, list of chemicals and materials with manufacturers and serial numbers. 


\section{References}

(1) Ardenkjaer-Larsen, J. H. On the present and future of dissolution-DNP. J. Magn. Reson. 2016, 264, 3 - 12, Hyperpolarized NMR Comes of Age.

(2) Nikolaou, P.; Goodson, B. M.; Chekmenev, E. Y. NMR Hyperpolarization Techniques for Biomedicine. Chem. Eur. J. 2015, 21, 3156-3166.

(3) Barskiy, D. A. et al. NMR Hyperpolarization Techniques of Gases. Chem. Eur. J. 2017, 23, 725-751.

(4) Comment, A. Dissolution DNP for in vivo preclinical studies. J. Magn. Reson. 2016, 264, 39 - 48, Hyperpolarized NMR Comes of Age.

(5) Kovtunov, K. V.; Pokochueva, E. V.; Salnikov, O. G.; Cousin, S. F.; Kurzbach, D.; Vuichoud, B.; Jannin, S.; Chekmenev, E. Y.; Goodson, B. M.; Barskiy, D. A.; Koptyug, I. V. Hyperpolarized NMR Spectroscopy: d-DNP, PHIP, and SABRE Techniques. Chem. As. J. 2018, 13, 1857-1871.

(6) Ardenkjaer-Larsen, J.-H. et al. Facing and Overcoming Sensitivity Challenges in Biomolecular NMR Spectroscopy. Angew. Chem. Int. Ed. 2015, 54, 9162-9185.

(7) Kim, Y.; Hilty, C. In Biological NMR Part $B$; Wand, A. J., Ed.; Methods in Enzymology; Academic Press, 2019; Vol. 615; pp $501-526$.

(8) Zhang, G.; Hilty, C. Applications of dissolution dynamic nuclear polarization in chemistry and biochemistry. Magn. Reson. Chem. 2018, 56, 566-582.

(9) Zhivonitko, V. V.; Kovtunov, K. V.; Skovpin, I. V.; Barskiy, D. A.; Salnikov, O. G.; Koptyug, I. V. Understanding Organometallic Reaction Mechanisms and Catalysis; John Wiley and Sons, Ltd, 2014; Chapter 7, pp 145-186.
(10) Kovtunov, K. V.; Zhivonitko, V. V.; Skovpin, I. V.; Barskiy, D. A.; Koptyug, I. V. In Hyperpolarization Methods in NMR Spectroscopy; Kuhn, L. T., Ed.; Springer Berlin Heidelberg: Berlin, Heidelberg, 2013; pp 123-180.

(11) Garcon, A.; Aybas, D.; Blanchard, J. W.; Centers, G.; Figueroa, N. L.; Graham, P. W.; Kimball, D. F. J.; Rajendran, S.; Sendra, M. G.; Sushkov, A. O.; Trahms, L.; Wang, T.; Wickenbrock, A.; Wu, T.; Budker, D. The cosmic axion spin precession experiment (CASPEr): a darkmatter search with nuclear magnetic resonance. Quantum Sci. Technol. 2017, 3, 014008.

(12) Wu, T.; Blanchard, J. W.; Centers, G. P.; Figueroa, N. L.; Garcon, A.; Graham, P. W.; Kimball, D. F. J.; Rajendran, S.; Stadnik, Y. V.; Sushkov, A. O.; Wickenbrock, A.; Budker, D. Search for Axionlike Dark Matter with a LiquidState Nuclear Spin Comagnetometer. Phys. Rev. Lett. 2019, 122, 191302.

(13) Jackson Kimball, D. F. et al. Overview of the Cosmic Axion Spin Precession Experiment (CASPEr). Microwave Cavities and Detectors for Axion Research. Cham, 2020; pp 105-121.

(14) Adams, R. W.; Aguilar, J. A.; Atkinson, K. D.; Cowley, M. J.; Elliott, P. I.; Duckett, S. B.; Green, G. G.; Khazal, I. G.; López-Serrano, J.; Williamson, D. C. Reversible interactions with para-hydrogen enhance NMR sensitivity by polarization transfer. Science 2009, 323, 1708-1711.

(15) Barskiy, D. A.; Knecht, S.; Yurkovskaya, A. V.; Ivanov, K. L. SABRE: Chemical kinetics and spin dynamics of the formation of hyperpolarization. Prog. Nucl. Magn. Reson. Spectrosc. 2019, 114-115, $33-70$.

(16) Farkas, A. Orthohydrogen, Parahydrogen 
and Heavy Hydrogen. Z. Elektrochem. Angew. Phys. Chem. 1935, 41, 812-812.

(17) Lloyd, L. S. et al. Hyperpolarisation through reversible interactions with parahydrogen. Catal. Sci. Technol. 2014, 4, 3544-3554.

(18) Barskiy, D. A.; Pravdivtsev, A. N.; Ivanov, K. L.; Kovtunov, K. V.; Koptyug, I. V. A simple analytical model for signal amplification by reversible exchange (SABRE) process. Phys. Chem. Chem. Phys. 2016, 18, 89-93.

(19) Barskiy, D. A.; Kovtunov, K. V.; Koptyug, I. V.; He, P.; Groome, K. A.; Best, Q. A.; Shi, F.; Goodson, B. M.; Shchepin, R. V.; Truong, M. L.; Coffey, A. M.; Waddell, K. W.; Chekmenev, E. Y. In Situ and Ex Situ Low-Field NMR Spectroscopy and MRI Endowed by SABRE Hyperpolarization. ChemPhysChem 2014, 15, 4100-4107.

(20) Cowley, M. J.; Adams, R. W.; Atkinson, K. D.; Cockett, M. C. R.; Duckett, S. B.; Green, G. G. R.; Lohman, J. A. B.; Kerssebaum, R.; Kilgour, D.; Mewis, R. E. Iridium N-Heterocyclic Carbene Complexes as Efficient Catalysts for Magnetization Transfer fromparaHydrogen. J. Am. Chem. Soc. 2011, 133, 6134-6137.

(21) Adams, R. W.; Duckett, S. B.; Green, R. A.; Williamson, D. C.; Green, G. G. R. A theoretical basis for spontaneous polarization transfer in non-hydrogenative parahydrogen-induced polarization. J. Chem. Phys. 2009, 131, 194505.

(22) Theis, T.; Truong, M. L.; Coffey, A. M.; Shchepin, R. V.; Waddell, K. W.; Shi, F.; Goodson, B. M.; Warren, W. S.; Chekmenev, E. Y. Microtesla SABRE enables $10 \%$ nitrogen-15 nuclear spin polarization. J. Am. Chem. Soc. 2015, 137, 1404-1407.
(23) Truong, M. L.; Theis, T.; Coffey, A. M.; Shchepin, R. V.; Waddell, K. W.; Shi, F.; Goodson, B. M.; Warren, W. S.; Chekmenev, E. Y. $15 \mathrm{~N}$ hyperpolarization by reversible exchange using SABRESHEATH. J. Phys. Chem. C 2015, 119, 8786-8797.

(24) Kiryutin, A. S.; Pravdivtsev, A. N.; Ivanov, K. L.; Grishin, Y. A.; Vieth, H.M.; Yurkovskaya, A. V. A fast field-cycling device for high-resolution NMR: Design and application to spin relaxation and hyperpolarization experiments. J. Magn. Reson. 2016, 263, $79-91$.

(25) Pravdivtsev, A. N.; Yurkovskaya, A. V.; Vieth, H.-M.; Ivanov, K. L. RF-SABRE: A Way to Continuous Spin Hyperpolarization at High Magnetic Fields. The journal of physical chemistry. $B$ 2015, 119, 13619-13629.

(26) Blanchard, J. W.; Wu, T.; Eills, J.; Hu, Y.; Budker, D. Zero- to ultralow-field nuclear magnetic resonance J-spectroscopy with commercial atomic magnetometers. J. Magn. Reson. 2020, 314, 106723.

(27) Put, P.; Pustelny, S.; Budker, D.; Druga, E.; Sjolander, T.; Pines, A.; Barskiy, D. Zero- to Ultralow-Field NMR Spectroscopy of Small Biomolecules. 2020 ,

(28) Colell, J. F. P.; Logan, A. W.; Zhou, Z.; Shchepin, R. V.; Barskiy, D. A.; Ortiz Jr, G. X.; Wang, Q.; Malcolmson, S. J.; Chekmenev, E. Y.; Warren, W. S., et al. Generalizing, extending, and maximizing nitrogen-15 hyperpolarization induced by parahydrogen in reversible exchange. $J$. Phys. Chem. C 2017, 121, 6626-6634.

(29) Truong, M. L.; Shi, F.; He, P.; Yuan, B.; Plunkett, K. N.; Coffey, A. M.; Shchepin, R. V.; Barskiy, D. A.; Kovtunov, K. V.; Koptyug, I. V.; Waddell, K. W.; Goodson, B. M.; Chekmenev, E. Y. Irreversible Catalyst Activation Enables Hyperpolarization 
and Water Solubility for NMR Signal Amplification by Reversible Exchange. J. Phys. Chem. B 2014, 118, 13882-13889, PMID: 25372972.

(30) Jalal, M.; Hammouti, B.; Touzani, R.; Aouniti, A.; Ozdemir, I. Metal-NHC heterocycle complexes in catalysis and biological applications: Systematic review. Mater. Today-Proc. 2020, 31, S122 S129.

(31) Kelly III, R. A.; Clavier, H.; Giudice, S.; Scott, N. M.; Stevens, E. D.; Bordner, J.; Samardjiev, I.; Hoff, C. D.; Cavallo, L.; Nolan, S. P. Determination of N-Heterocyclic Carbene (NHC) Steric and Electronic Parameters using the $[(\mathrm{NHC}) \operatorname{Ir}(\mathrm{CO}) 2 \mathrm{Cl}]$ System. Organometallics 2008, 27, 202-210.

(32) Vazquez-Serrano, L. D.; Owens, B. T.; Buriak, J. M. The search for new hydrogenation catalyst motifs based on $\mathrm{N}$ heterocyclic carbene ligands. Inorganica Chim. Acta 2006, 359, 2786 - 2797, Protagonists in Chemistry: Brian James.

(33) Savka, R.; Plenio, H. Facile synthesis of [(NHC)MX(COD)] and $\left[(\mathrm{NHC}) \mathrm{MCl}(\mathrm{CO})_{2}\right] \quad(\mathrm{M}=\mathrm{Rh}, \quad \mathrm{Ir} ; \mathrm{X}$ $=\mathrm{Cl}, \mathrm{I})$ complexes. Dalton Trans. 2015, 44, 891-893.

(34) Blanchard, J. W.; Budker, D. Zero to Ultralow Field NMR. eMagRes 2016, 5, 1395-1410.

(35) Ledbetter, M.; Theis, T.; Blanchard, J.; Ring, H.; Ganssle, P.; Appelt, S.; Blümich, B.; Pines, A.; Budker, D. Nearzero-field nuclear magnetic resonance. Physical review letters 2011, 10\%, 107601.

(36) Tayler, M. C.; Sjolander, T. F.; Pines, A.; Budker, D. Nuclear magnetic resonance at millitesla fields using a zero-field spectrometer. Journal of Magnetic Resonance 2016, 270, 35-39.
(37) Theis, T.; Ledbetter, M. P.; Kervern, G.; Blanchard, J. W.; Ganssle, P. J.; Butler, M. C.; Shin, H. D.; Budker, D.; Pines, A. Zero-field NMR enhanced by parahydrogen in reversible exchange. $J$. Am. Chem. Soc. 2012, 134, 3987-3990.

(38) Zaccari, D. G.; Snyder, J. P.; Peralta, J. E.; Taurian, O. E.; Contreras, R. H.; Barone, V. Natural $J$-coupling analysis of the electron lone pair effect on NMR couplings: 2. The anomeric effects on ${ }^{1} J(\mathrm{C}, \mathrm{H})$ couplings and its dependence on solvent. Mol. Phys. 2002, 100, 705715 .

(39) Zaccari, D.; Barone, V.; Peralta, J. E.; Contreras, R. H.; Taurian, O. E.; Diez, E.; Esteban, A. Solvent Effects on Nuclear Magnetic Resonance ${ }^{2} \mathrm{~J}(\mathrm{C}, \mathrm{H})$ and ${ }^{1} \mathrm{~J}(\mathrm{C}, \mathrm{H})$ Spin-Spin Coupling Constants in Acetaldehyde. Int. J. Mol. Sci. 2003, 4, 93106. 


\section{Graphical TOC Entry}

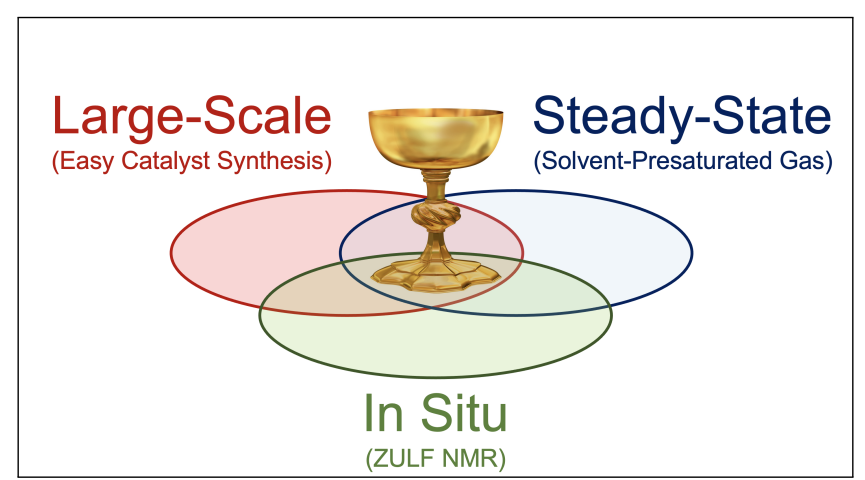

